\title{
Resonance coupling induced enhancement of indirect transverse cooling in a laser-cooled ion beam
}

\author{
M. Nakao, ${ }^{*}$ T. Hiromasa, ${ }^{\dagger}$ H. Souda, M. Tanabe, ${ }^{\ddagger}$ T. Ishikawa, ${ }^{\S}$ H. Tongu, and A. Noda \\ ICR, Kyoto University, Uji-city, Kyoto, Japan \\ K. Jimbo \\ IAE, Kyoto University, Uji-city, Kyoto, Japan \\ T. Shirai \\ NIRS, Chiba-city, Chiba, Japan \\ M. Grieser \\ MPI-K, Heidelberg, Germany \\ H. Okamoto \\ Graduate School of Advanced Science of Matter, Hiroshima University, Higashi-Hiroshima-city, Hiroshima, Japan
}

\author{
A. V. Smirnov \\ JINR, Dubna, Moscow Region, Russia \\ (Received 28 October 2011; published 30 November 2012)
}

\begin{abstract}
We report the first observation of indirect resonantly enhanced transverse laser cooling of a bunched, stored ion beam of $40 \mathrm{keV}^{24} \mathrm{Mg}^{+}$. The longitudinal velocity distribution and the horizontal beam size of the laser-cooled ${ }^{24} \mathrm{Mg}^{+}$ion beam with $280 \mathrm{~nm}$ laser light were simultaneously observed by the use of standard fluorescence-based techniques. Keeping the operation point at $(2.068,1.105)$, the synchrotron tune $\left(\nu_{s}\right)$ was changed from 0.0376 to 0.1299 . A strong decrease in the cooled horizontal beam size and a corresponding increase in the equilibrium cooled momentum spread were observed at the expected synchrobetatron resonance coupling condition of $\nu_{s}=0.068$, which is the first observation of a resonantly induced coupling for enhanced indirect transverse laser cooling of an ion beam, and is an important step towards creating a crystalline ground state of the beam.
\end{abstract}

DOI: 10.1103/PhysRevSTAB.15.110102

PACS numbers: 29.27.Fh, 29.27.Bd, 29.27.Eg

\section{INTRODUCTION}

The temperature of a stored and circulating beam in a storage ring can be reduced by conventional methods such as electron beam cooling, invented by Budker [1], and stochastic cooling, invented by Van der Meer [2]. Another relatively new method is Doppler laser cooling originally developed for stationary atoms $[3,4]$ and later applied for circulating ion beams. Among these methods, laser cooling is considered the most promising to achieve the lowest temperature, due to its very strong cooling force. The first

\footnotetext{
*nakao@kyticr.kuicr.kyoto-u.ac.jp

Present address: Mitsubishi Electric Corporation, Amagasaki-city, Hyogo, Japan.

${ }^{\ddagger}$ Present address: NHK, Kitakyushu-city, Fukuoka, Japan.

${ }^{\S}$ Present address: Shimadzu Corporation, Kyoto-city, Kyoto, Japan.
}

Published by the American Physical Society under the terms of the Creative Commons Attribution 3.0 License. Further distribution of this work must maintain attribution to the author(s) and the published article's title, journal citation, and DOI. laser-cooling experiment of an ion beam was carried out at Test Storage Ring (TSR) by Schröder et al. [5], and followed at Aarhus Storage Ring in Denmark (ASTRID) by Hangst et al. [6]. Both groups successfully cooled the longitudinal motion of coasting beams of heavy ions circulating at high speed in the laboratory frame; this technique was further extended for a bunched beam also in the ASTRID ring by Hangst et al. [7].

In order to ensure a sufficient spatial overlap between photons and moving ions, a cooling laser, either copropagating or counterpropagating with the ion beam, is introduced along a straight section of a ring. Then, the momentum transfer from the photons to the ions occurs only longitudinally, which means no direct cooling is expected in the transverse directions. Later, Miesner et al. found that the transverse motion is sympathetically coolable via intrabeam scattering (IBS) [8], but the sympathetic cooling efficiency was not very high at an ordinary particle density. As pointed out by Madsen et al. [9], IBS-induced sympathetic cooling may cease to be sufficiently strong when the beam gets cold enough to overcome heating by the spontaneous emitted photons, as well as by ring 

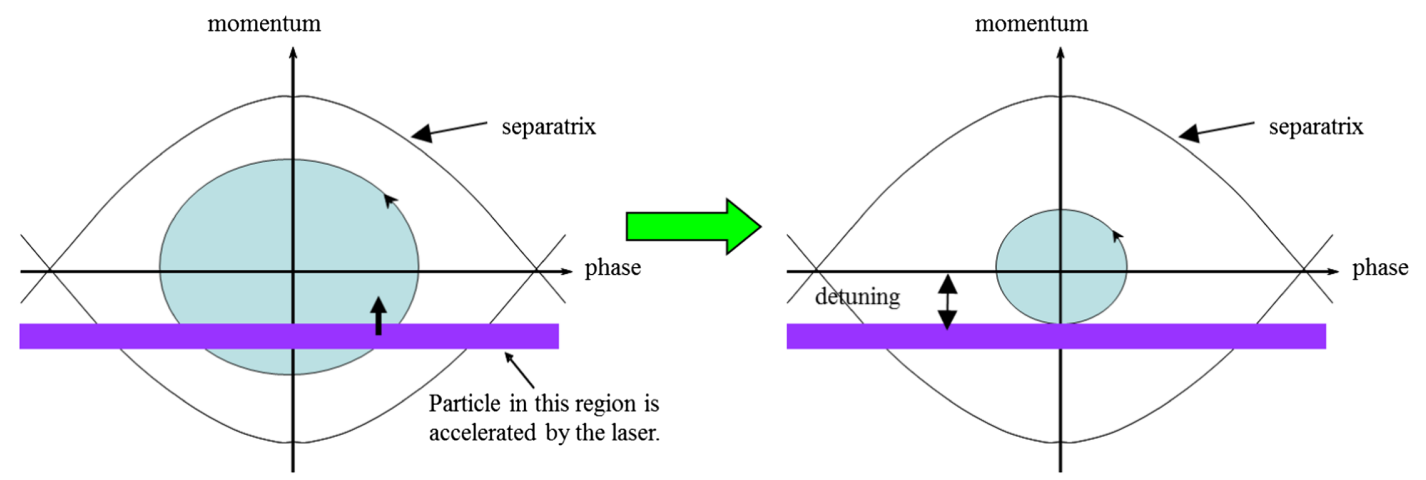

FIG. 1. Schematic diagram of Doppler laser cooling of a bunched beam. Each ion circulates around the origin in the longitudinal phase space at the frequency determined by the synchrotron tune. Ions with large synchrotron-oscillation amplitudes periodically come into the laser-cooling band and absorb and emit many photons. Since the laser is copropagating with the beam in S-LSR, the ions are accelerated after photon absorption, which effectively reduces the synchrotron amplitudes. As a result, the beam is compressed in the phase plane as illustrated in the right picture.

imperfections. The sympathetic scheme is thus irrelevant for our present purpose to achieve fast multidimensional laser cooling and consequent ultracold beam generation. In order to overcome these difficulties, we employed the synchrobetatron resonance coupling (SBRC) scheme, proposed by Okamoto et al. [10,11], to artificially and dramatically enhance the transverse indirect laser-cooling efficiency. According to molecular dynamics (MD) simulations by Wei et al. [12], it is theoretically possible to reduce the beam temperature in all three dimensions if the cooling force is sufficiently strong, and the ring lattice is properly designed to satisfy the so-called "maintenance condition." In the SBRC scheme, the operating point of a cooler storage ring is set on a linear difference resonance condition where the betatron tunes $\left(\nu_{x}, \nu_{y}\right)$ and the synchrotron tune $\nu_{s}$ fulfill

$$
\begin{aligned}
& \nu_{s}-\nu_{x}=\text { integer, } \\
& \nu_{x}-\nu_{y}=\text { integer. }
\end{aligned}
$$

Under the condition that Eq. (1) is satisfied and a radiofrequency (rf) cavity is located at a position with finite dispersion, the longitudinal degree of freedom couples with the horizontal one. Linear coupling between the horizontal and vertical directions can be provided under the condition of Eq. (2) either by a solenoid or by a skew quadrupole magnet. The powerful longitudinal cooling effect is effectively transferred through these coupling sources under the resonance conditions and we can thus expect efficient, indirect laser cooling in both transverse directions. Lauer et al. have demonstrated another indirect transverse cooling scheme based on a dispersive transverse cooling force caused by horizontal displacement of a cooling laser [13]. This scheme, however, has a practical limitation that the laser power is not fully utilized for beam cooling because of a poor overlap between the laser and ions.

At ESR, laser cooling of highly relativistic ${ }^{12} \mathrm{C}^{3+}$ ions has been performed at $1.47 \mathrm{GeV}$ [14]. It is also worth noting that the coasting crystalline string of ${ }^{24} \mathrm{Mg}^{+}$ions with the use of laser cooling was experimentally realized for energy of $1 \mathrm{eV}$ at the tabletop circular ion trap, PALLAS [15], which was also further extended for a bunched beam [16].

Taking the above-mentioned historical achievements into account, we designed and constructed the small laser-equipped storage ring (S-LSR) for the demonstration of efficient multidimensional laser cooling $[17,18]$. For the past several years, continuous effort has been devoted to attain a three-dimensionally ultracold ion beam circulating in a storage ring. In our present study, we more or less follow the ASTRID approach where a single laser is employed together with an rf voltage to cool a bunched ${ }^{24} \mathrm{Mg}^{+}$ ion beam in the longitudinal direction [7]. In case a beam is bunched with an rf field, individual ions execute synchrotron oscillations in the longitudinal phase space, and the rf electric field works as a counter force to the ions in the beam accelerated by copropagating laser with Doppler laser cooling, as shown in Fig. 1. The closed orbit distortion (COD) in the laser-cooling section is precisely corrected by the use of correction coils in the dipole magnets (horizontal direction) and steering electrodes (vertical direction). The injection orbit and COD were optimized to overlap the beam and the laser [19]. The first laser-cooling experiment at S-LSR was carried out several years ago with a coasting beam utilizing an induction deceleration as the counter force of laser-photon acceleration, where the longitudinal temperature was reduced to $3.6 \mathrm{~K}$ at the equilibrium. IBS coupling between the longitudinal and vertical degrees of freedom resulted in a vertical temperature of $500 \mathrm{~K}$ (Tanabe et al. [20]). We then recently made a step forward to improve transverse cooling efficiency employing the SBRC scheme for a bunched beam [11]. This paper addresses the first experimental observation of indirect horizontal laser cooling at S-LSR. After outlining the experimental procedure in Sec. II, we give in Sec. III clear evidence that the horizontal cooling efficiency is actually enhanced under the resonance condition of Eq. (1). 


\section{EXPERIMENTAL PROCEDURE}

\section{A. Small laser-equipped storage ring, S-LSR}

The layout of the S-LSR with a laser cooling and observation systems for a $40 \mathrm{keV}{ }^{24} \mathrm{Mg}^{+}$ion beam is shown in Fig. $2 .{ }^{24} \mathrm{Mg}^{+}$ions, accelerated by a high electrostatic voltage at a sputter ion source (DANFYSIK CHORDIS model 921) is transported through the beam-transport line, and single-turn injected into the S-LSR ring with the use of a kicker magnet. The ion beam is overlapped with a laser light in one of the six straight sections, where the beamcollimation apertures and a post-acceleration tube (PAT), described later, are installed. The principal parameters of the S-LSR and the cooling laser for the present experiment are listed in Table I.

\section{B. Laser-cooling system}

\section{Laser generating and transfer system}

In the present experiment, a single laser system is utilized for both cooling and observing the beam characteristics. An overall view of the laser system is shown in Fig. 3. A solid state laser (a) (Coherent Verdi V-10) (wavelength of $532 \mathrm{~nm}$; usually we operate at a power of $6.6 \mathrm{~W}$ ) pumps a ring dye laser (b) (CR-699-21) employing rhodamine 560 chloride $(560 \mathrm{~nm} ; 600 \mathrm{~mW})$. The frequency of the output cooling laser can be controlled by tuning the frequency of the dye laser. A second-harmonic generator (c) with a BBO nonlinear optical crystal (Coherent MBD200) $(280 \mathrm{~nm} ; 50 \mathrm{~mW})$ doubles the frequency of the dye laser, producing an ultraviolet laser light for the experiment. The dye laser is stabilized by a feedback system, which suppresses the frequency deviation within $10 \mathrm{MHz}$ in one injection. The root-mean-square drift of the dye laser frequency over a long time period is less than $20 \mathrm{MHz} / \mathrm{min}$. The laser spot size at the CCD observation

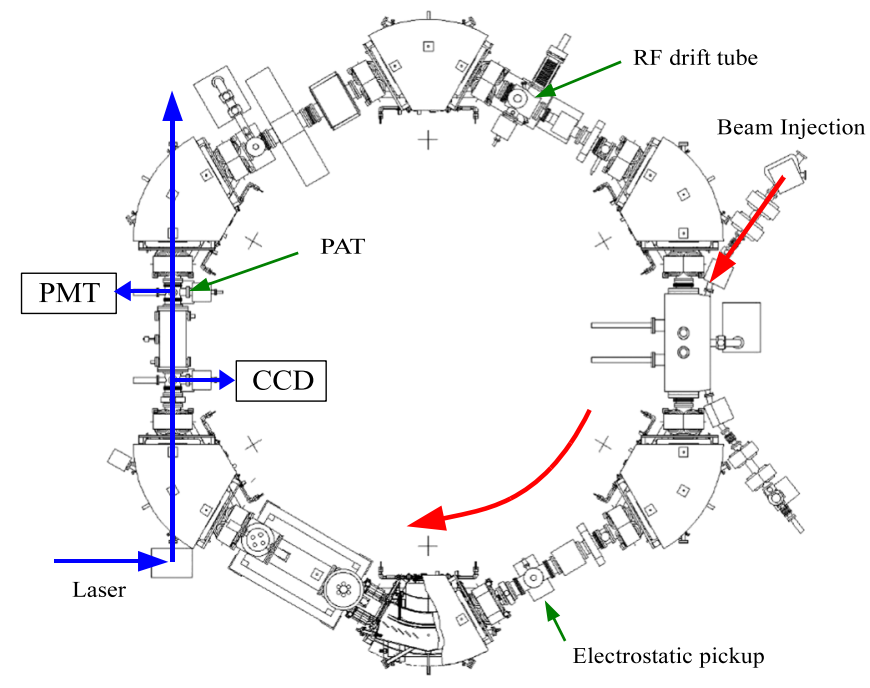

FIG. 2. Layout of S-LSR and equipment for laser-cooling experiments.
TABLE I. Parameters of laser cooling at S-LSR.
Circumference

Average radius

Length of straight section

Radius of curvature

Super periodicity

Ion species

Natural width of ${ }^{24} \mathrm{Mg}^{+}$

Initial momentum spread

Initial emittance

Initial particle number

Betatron tune

Synchrotron tune

Laser frequency

Detuning

Laser power at exit window

Laser spot size $(1 \sigma)$

\section{$22.557 \mathrm{~m}$}

$3.59 \mathrm{~m}$

$1.05 \mathrm{~m}$

6

${ }^{24} \mathrm{Mg}^{+}: 40 \mathrm{keV}$

$2 \pi \times 42.7 \mathrm{MHz}$

$1 \times 10^{-3}$

$(6.8 \pi, 2.4 \pi) \mathrm{mm} \mathrm{mrad}$

$3 \times 10^{7}$

$(2.068,1.105)$

$0.0376-0.1299$

$1074110.3 \mathrm{GHz} \pm 0.01 \mathrm{GHz}$

$-0.2 \mathrm{GHz} \pm 0.01 \mathrm{GHz}$

$7-15 \mathrm{~mW}$

$1.0 \mathrm{~mm}$
$1.86 \mathrm{~m}$

section is $1.0 \mathrm{~mm}$ in $1 \sigma$. The laser spot is made roughly circular by a pinhole, a collimator, and lenses. The obtained laser power for beam cooling is in the range of 7 to $15 \mathrm{~mW}$.

${ }^{24} \mathrm{Mg}^{+}$ions are excited from the ground $3 \mathrm{~s}^{2} \mathrm{~S}_{1 / 2}$ state to the excited $3 \mathrm{p}^{2} \mathrm{P}_{3 / 2}$ state by absorption of laser photons. Ions in the upper state quickly return to the original state spontaneously emitting photons, thus moving back and forth between the two levels. The lifetime of the excited state is $3.7 \mathrm{~ns}$ and the natural width of this cooling transition is $2 \pi \times 42.7 \mathrm{MHz}$. It determines the Doppler limit of the possible lowest temperature of the cooled beam. In the actual experiment, we fixed the detuning of the laser frequency at $0.2 \mathrm{GHz}$ (corresponding to the fractional momentum difference, $\Delta p / p=1.0 \times 10^{-4}$ ) and did not sweep the frequency due to technical reasons. The initial momentum spread of $1 \times 10^{-3}(1 \sigma)$ was then reduced to

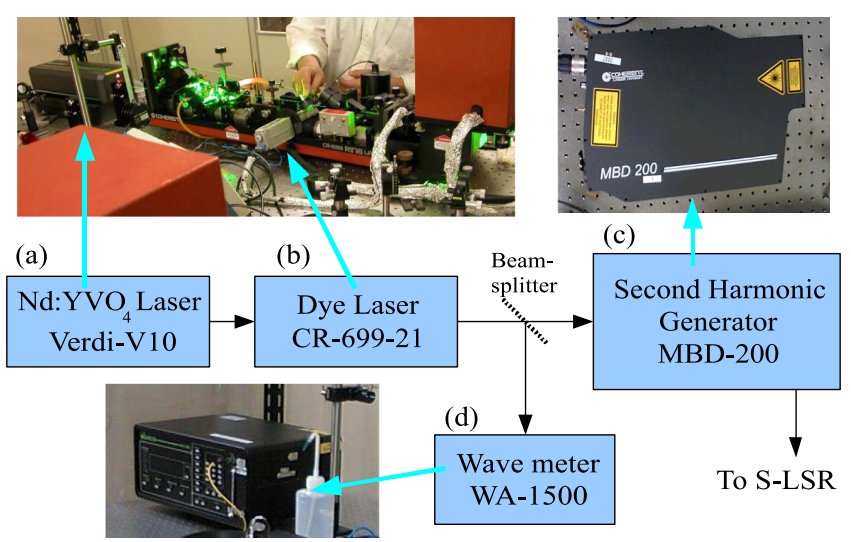

FIG. 3. Main components of the laser system. (a) A solid state laser with the frequency of $532 \mathrm{~nm}$, (b) a $560 \mathrm{~nm}$ ring dye laser pumped by the solid state laser, (c) a $280 \mathrm{~nm}$ second-harmonic generator using a BBO nonlinear optical crystal and (d) a wavelength meter, which has a precision of $0.01 \mathrm{GHz}$ 


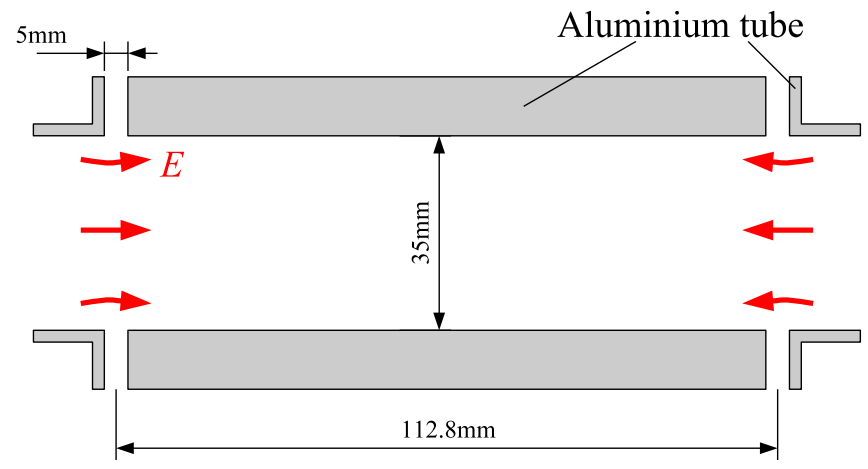

FIG. 4. Schematic drawing of the double-gap drift-tube cavity for SBRC experiments at S-LSR. The cavity is designed to bunch a $40 \mathrm{keV}{ }^{24} \mathrm{Mg}^{+}$beam at the harmonic number of 100 . The direction of the $\mathrm{rf}$ electric field is reversed in every half $\mathrm{rf}$ period.

$1.3 \times 10^{-4}(1 \sigma)$ for a bunched beam as described below. In the SBRC scheme, the synchrotron tune has to be finite due to the condition in Eq. (1); the beam is, therefore, necessarily bunched. Since the beam is always coasting at injection in S-LSR, we need to adiabatically capture ions within the rf buckets, increasing the rf amplitude from zero to a certain final value. Although the laser is always on at a fixed frequency throughout the experiment, its effect on the beam should be weak during the initial adiabatic capturing stage because only a limited fraction of stored ions resonate with laser photons. Once the rf buckets are formed, all ions circulate around the origin of the longitudinal phase plane, and then laser cooling starts effectively.

\section{Synchrobetatron coupling}

A compact drift-tube cavity with two accelerating gaps is particularly developed for the SBRC experiments. It is placed in a straight section (see Fig. 2) to bunch the beam at the harmonic number of 100 . The geometry of the drift tube and the direction of the applied rf electric field are shown in Fig. 4. The distance between the centers of the two gaps is $113 \mathrm{~mm}$, so that the phase advance for a $40 \mathrm{keV}{ }^{24} \mathrm{Mg}^{+}$ion beam is $\pi$. The ring dispersion at the cavity position is $1.1 \mathrm{~m}$, which yields the linear longitudinal-horizontal coupling required for indirect transverse cooling. As mentioned above, the beam is adiabatically captured and fully bunched within 5000 turns $(0.2 \mathrm{sec}$.) after injection. The horizontal and vertical betatron tunes are set to be 2.068 and 1.105 , respectively. On the other hand, the synchrotron tune is varied over a wide range by changing the rf voltage of the cavity. Figure 5(a) shows a block diagram of our betatron tune measurement system using the beam transfer function. The beam is kicked by a pair of rf knock out

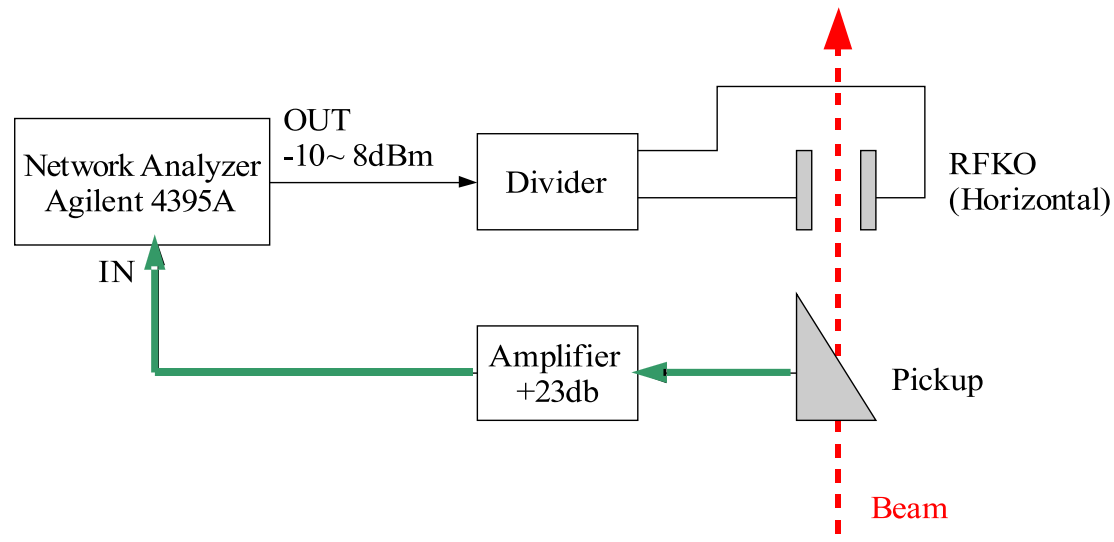

(a)

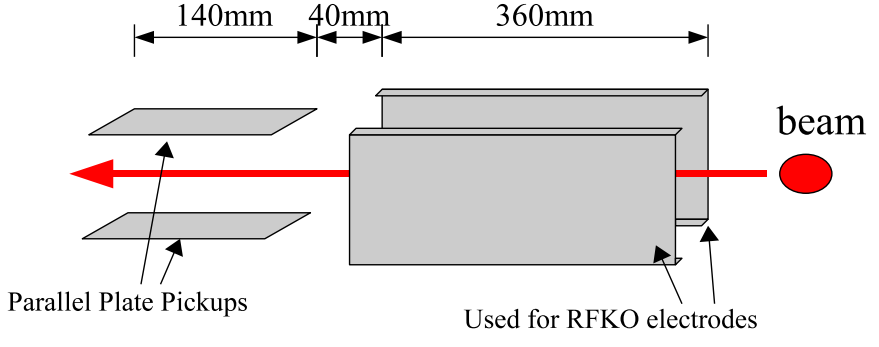

Side View

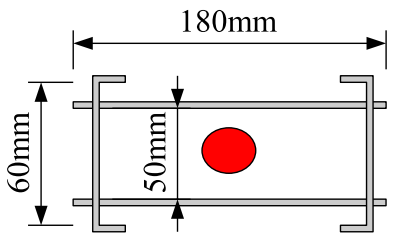

Cross Sectional View

FIG. 5. (a) Block diagram of the betatron tunes and the synchrotron-tune measurement system. A small perturbation is applied to the beam with RFKO. If the applied perturbation frequency is near the synchrotron sideband, the beam fluctuation signal is enhanced, and it is detected with a triangle pickup. (b) Schematic drawing of the horizontal RFKO electrodes and "Parallel Plate Pickups" to measure the beam intensity. 
(RFKO) electrodes [see Fig. 5(b)] excited by a network analyzer (Agilent 4395A). The transverse and longitudinal beam oscillations are observed by a plate pickup of triangular shape, as indicated by green arrows [21]. Since a pickup signal without the excitation of RFKO is free from the betatron motion, we can separate the synchrotronoscillation component, which enables us to determine all three tunes.

The number of particles circulating in the storage ring and the beam lifetime are measured from bunched-beam signals induced on a pair of parallel-plate electrostatic pickups [Fig. 5(b)] [22]. The rf synchronous phase is set at $\phi_{s}=0$ where the beam is neither accelerated nor decelerated but only bunched. The synchrotron tune, $\nu_{s}$, is then given by

$$
\nu_{s}=\sqrt{\frac{h|\eta| e V_{\text {eff }}}{2 \pi \beta^{2} E_{0}}}
$$

where $h$ is the harmonic number, $\beta$ and $\gamma$ are the Lorentz factors, $\eta$ is the phase slip factor (defined as $1 / \gamma_{T}^{2}-1 / \gamma^{2}$ with $\gamma_{T}$ being the transition gamma of the ring), $e$ is the electron charge magnitude, $V_{\text {eff }}$ is the effective rf voltage, and $E_{0}$ is the total energy of the synchronous particle. The effective voltage is given by $V_{\text {eff }}=2 T V$, where $T$ and $V$ are the transit time factor ( 0.88 in our two-gap cavity) and the cavity voltage, respectively. The fractional parts of the measured betatron and synchrotron tunes are plotted in Fig. 6 as functions of the rf voltage. Both horizontal and synchrotron tunes jump slightly at the rf voltage of around $30 \mathrm{~V}$, where the synchrobetatron resonance condition in Eq. (1) is satisfied [22].

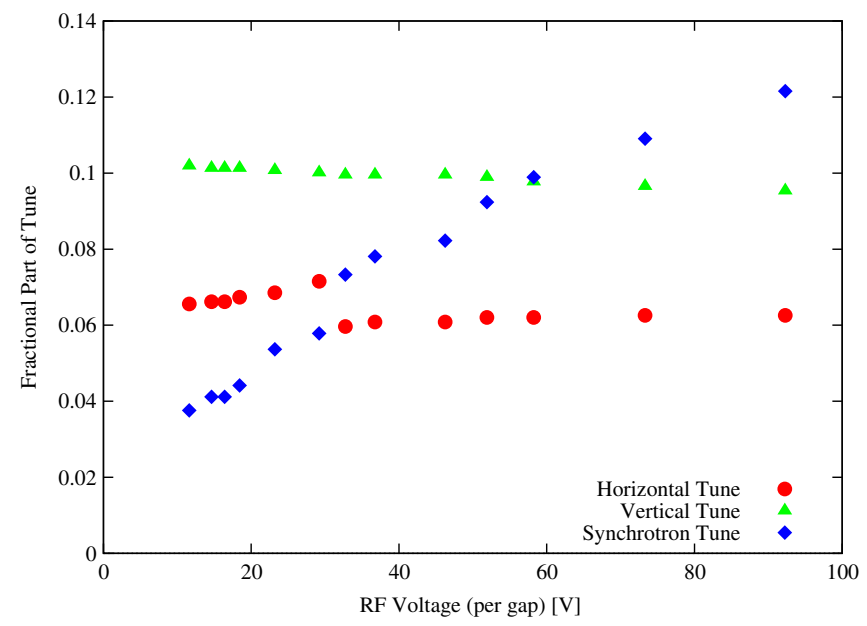

FIG. 6. Results of tune measurements. The fractional parts of the horizontal, vertical, and synchrotron tunes are plotted as functions of the rf cavity voltage. The integer parts of the horizontal and vertical tunes are 2 and 1, respectively. The distance between the horizontal and synchrotron tunes on coupling resonance $(\sim 30 \mathrm{~V})$ depends on the coupling strength.

\section{Longitudinal momentum-spread measurement with the PAT}

The momentum distribution of a circulating beam is measured by sweeping the beam energy with an electrostatic potential on a cylindrical electrode, whose axis is set parallel to the designed beam line. Such an apparatus was utilized at TSR first [23], and further developed by the ASTRID group [24] who referred it to as PAT [25]. A PAT, electrically insulated from the vacuum chamber by two ceramic spacers, is installed in the cooling section of the S-LSR. The PAT is vertically movable with a bawl screw and a pulse motor [Fig. 7(a)] and is electrically connected to a function generator so that we can apply an arbitrary timedependent voltage. The inner diameter and length of the cylinder are 35 and $44 \mathrm{~mm}$, respectively. A circular window of $10 \mathrm{~mm}$ in diameter is located on one side, which is utilized for spontaneous emission observations [Fig. 7(b)]. According to $3 \mathrm{D}$ numerical calculations, the fringe field does not seriously affect the potential uniformity in the region observed through the side window. The estimated potential distortion near the window is less than $0.5 \mathrm{~V}$ for the maximum applied voltage of $100 \mathrm{~V}$. Since the energy of each individual particle in the PAT is slightly modified by the electrical potential, a sweep of the PAT voltage results in a sweep of the particle velocity. This controlled velocity

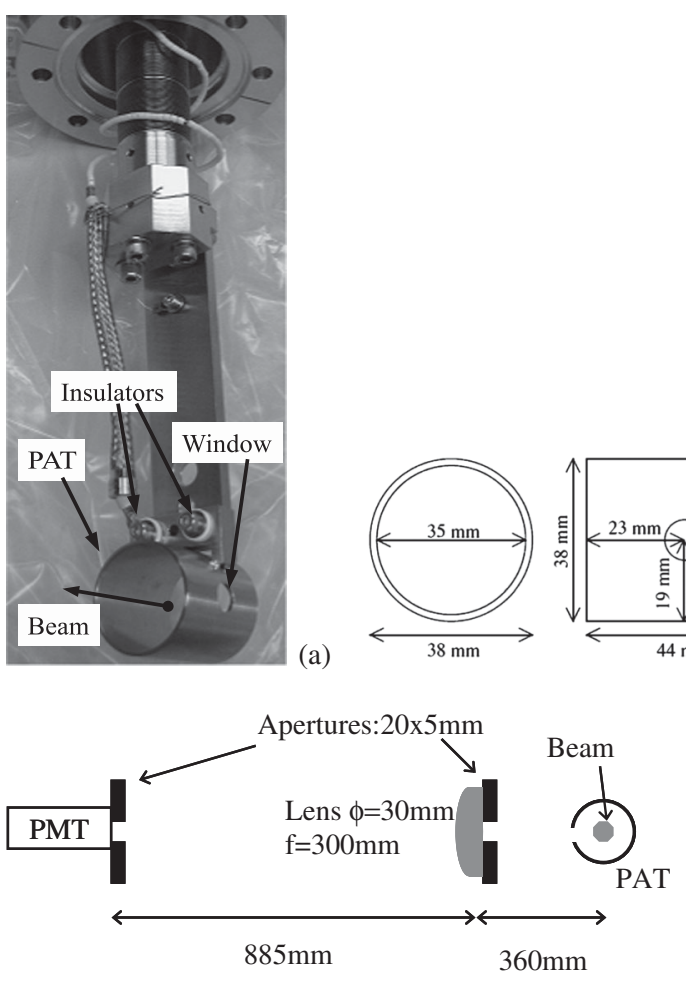

(c)

FIG. 7. The PAT system employed for momentum distribution measurements in S-LSR. (a) A photograph of the PAT and its support. The PAT is vertically inserted toward the beam (see Fig. 2) in the cooling section. (b) The geometry of the PAT. (c) The layout of the PAT system. 


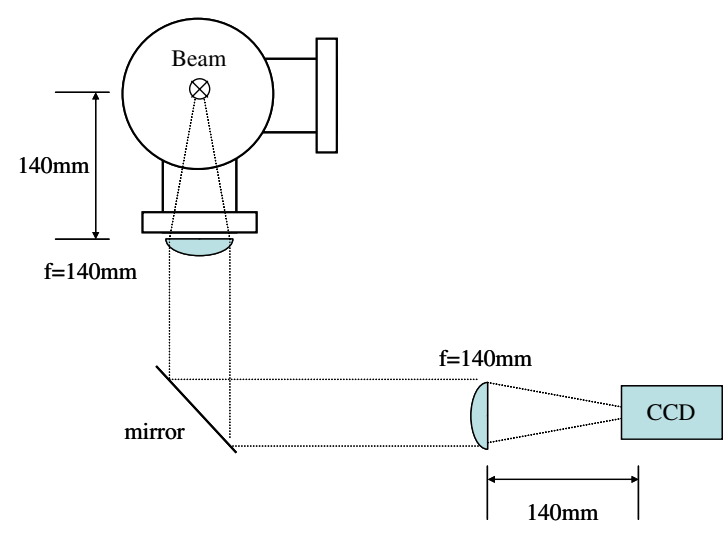

FIG. 8. A rough sketch of the horizontal beam-size measurement system. The main components of the system are a CCD camera, two focusing lenses, and one mirror.

sweep enables us to figure out the longitudinal momentum distribution of the beam from the fluorescence intensity because fluorescence photons are emitted only from ions with a proper Doppler shift. The sweep range is chosen wide enough to cover the velocity spread of the whole beam.

The variation range of the PAT voltage is $100 \mathrm{~V}$ corresponding to the energy difference of $2.5 \times 10^{-3}$ for a $40 \mathrm{keV}{ }^{24} \mathrm{Mg}^{+}$ion beam. The fluorescence photons through the side window of the PAT are detected with a photomultiplier tube (PMT, Hamamatsu Photonics R7056) [Fig. 7(c)]. A PMT signal is fitted with a Gaussian curve, dealing with the noise due to the reflected laser light from the vacuum chamber or the PAT as a pedestal, then we can figure out the beam momentum spread. The initial momentum spread estimated in this way was $1 \times 10^{-3}$, in agreement with the measurement of the coasting beam just after injection utilizing the well-known Schottky signal measurement [26]. After laser cooling, this number was decreased to $1.3 \times 10^{-4}$ in $1 \mathrm{sec}$ by the laser cooling. In the present experiment, the energy sweep by the PAT was initiated at beam injection and continued for $1 \mathrm{sec}$.

\section{Optical measurement of the horizontal beam profile}

The horizontal beam size was observed by CCD imaging. Spontaneous emission from the exited level of ${ }^{24} \mathrm{Mg}^{+}$ ions is focused into a parallel light by a lens that has a focal length of $140 \mathrm{~mm}$ and is set below the designed beam orbit. The parallel light is deflected by 90 degrees with a mirror and focused by another lens with the same focal length. The CCD camera (Hamamatsu Photonics C7190-11W) is placed $140 \mathrm{~mm}$ behind the lens as illustrated in Fig. 8, which means that the CCD image has the same size as the actual beam size.

In order to calibrate the scale of CCD images, we inserted a pinhole of $2 \mathrm{~mm} \phi$ into the beam line. The size of the pinhole image was 112 pixels on the CCD, which indicates that a single pixel corresponds to $2 / 112=$ $0.0179 \mathrm{~mm}$. An example of a typical beam image detected by the CCD camera is shown in Fig. 9(a). The abscissa and the ordinate correspond to the longitudinal and horizontal directions, respectively. Integrating the signal within the closed region surrounded by a red line along the longitudinal direction (abscissa), we obtained the horizontal beam profile as shown in Fig. 9(b).

\section{EXPERIMENTAL RESULTS AND DISCUSSION}

Figure 10 shows the time evolution of horizontal beam images measured at three different synchrotron tunes (rf voltages). As shown in Fig. 10, the horizontal image size increases due to IBS heating at a synchrotron tune of 0.038 (green points) where no SBRC is activated. By

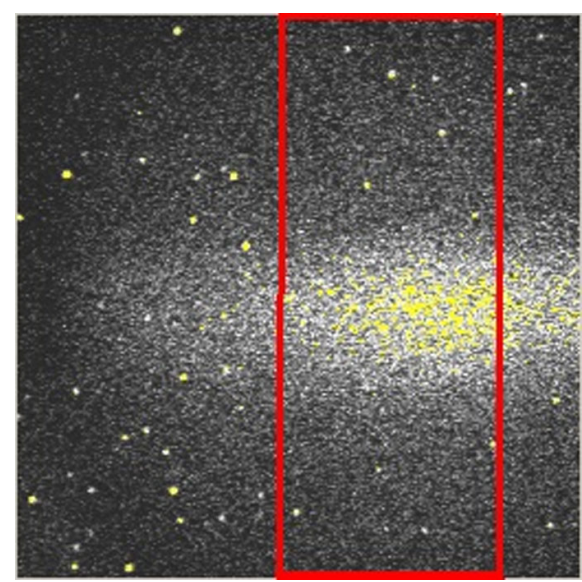

(a)

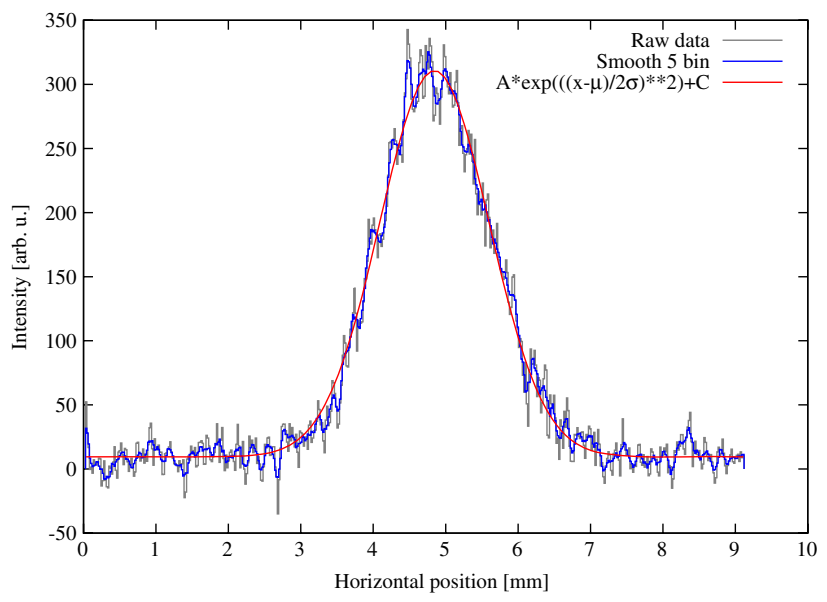

(b)

FIG. 9. Typical results of horizontal beam-size measurements with the CCD camera. (a) Fluorescence image of a ${ }^{24} \mathrm{Mg}^{+}$beam after injection. In this picture, the abscissa represents the direction of beam propagation. (b) The horizontal beam profile evaluated by integrating the fluorescence data (a) within the red rectangular region. 


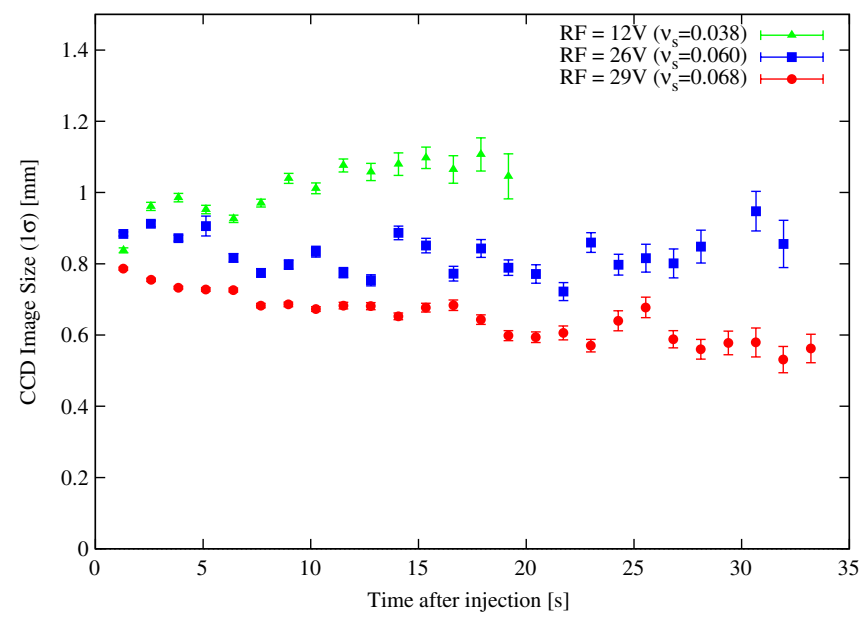

FIG. 10. Time evolution of the horizontal beam size at three different synchrotron tunes, i.e. $\nu_{s}=0.038,0.060$, and 0.068 . When the linear coupling resonance is excited $\left(\nu_{s}=0.068\right)$, the CCD image size in the horizontal direction continuously decreases from $\sim 1 \mathrm{~mm}(1 \sigma)$ to $0.55 \mathrm{~mm}(1 \sigma)$, which means that the horizontal beam temperature is reduced to $\sim 200 \mathrm{~K}$ by indirect laser cooling.

contrast, a reduction of the beam-image size is observed on the condition that the synchrotron tune is adjusted to the optimum resonant value 0.068 (red points) satisfying Eq. (1). At an intermediate tune of 0.060 (blue points), the beam-image size is roughly maintained, which suggests that the indirect horizontal laser-cooling force via SBRC balances with the IBS heating force. The synchrotron-tune dependence of the horizontal beamimage size after $30 \mathrm{sec}$ from the injection is plotted in Fig. 11 with blue triangles. Although there might be a possibility that the measured image size of $\sim 1 \mathrm{~mm}$ in the off-resonant region is underestimated due to the limited laser spot size, the CCD image near the synchrobetatron coupling is much smaller than the laser spot. We can thus conclude that the beam was actually compressed to the observed size in the horizontal direction under the SBRC condition. It is informative to note that, in Fig. 11, the momentum spread is maximized to be $1.5 \times 10^{-4}$ near the coupling resonance. This is due to the energy transfer from the horizontal direction to the longitudinal via SBRC. The momentum spread $\Delta p / p$ can directly be related to the longitudinal temperature $T_{L}$ as $k_{B} T_{L}=m c^{2} \beta^{2}(\Delta p / p)^{2}$, where $k_{B}$ and $m$ are the Boltzmann constant and the mass of the ion, respectively. This formula gives $21 \mathrm{~K}$ for the peak longitudinal beam temperature on resonance, which gradually decreases to about $14 \mathrm{~K}$ in the offresonant region.

At present, we cannot simultaneously measure the vertical beam size because only a single CCD camera is available. It is difficult to study a possible vertical heating mechanism in a laser-cooled, low-current beam, reported by Madsen et al. [9]. Previous experiments performed a few years ago at S-LSR with a different diagnostic setup,

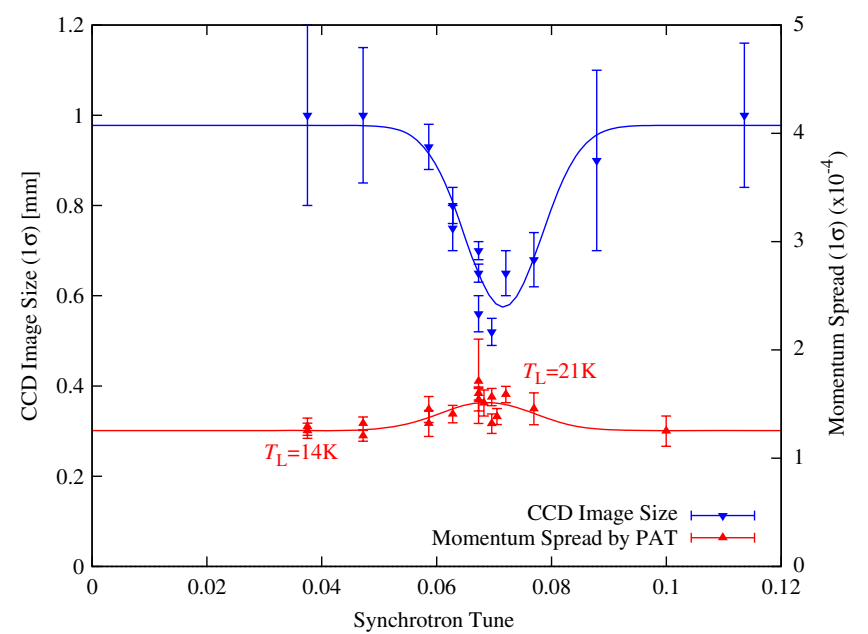

FIG. 11. Synchrotron-tune dependence of the horizontal beam size measured at $30 \mathrm{sec}$ after injection by the CCD camera. The longitudinal momentum spread measured with the PAT $1 \mathrm{sec}$ after injection is plotted in red triangles as a function of the synchrotron tune. The betatron tunes are fixed at $\left(\nu_{x}, \nu_{y}\right)=$ $(2.068,1.105)$ in this experiment. On the coupling resonance given by Eq. (1), the horizontal temperature goes down to $\sim 200 \mathrm{~K}$, and the longitudinal temperature goes up to $21 \mathrm{~K}$ from $14 \mathrm{~K}$ by the heat transfer from the horizontal degree of freedom. Note that, because of the limited laser spot size $(\sim 1 \mathrm{~mm})$, the observed beam-image size may be underestimated in the region at off resonance.

however, indicated no such vertical instability. This might be due to a large discrepancy in beam current between the Madsen's experiment and ours. In fact, our experiment was carried out with $1 \times 10^{7}$ to $3 \times 10^{7}$ ions stored in S-LSR and the beam was bunched. Madsen et al. observed a sudden vertical beam blowup when the number of ${ }^{24} \mathrm{Mg}^{+}$ ions in ASTRID was reduced to the order of $10^{5}$. This total particle number corresponds to a beam line density much lower than that of our case.

Another possible heating source is the space-chargeinduced linear resonance theoretically predicted by Wei et al. [12] and experimentally confirmed by Madsen et al. [27]. The linear collective resonance is unavoidable whenever the betatron phase advance per single lattice period of a ring exceeds 90 degrees. ${ }^{1}$ The limitations from strong tune shifts may have to be overcome to realize the crystalline string as discussed by Madsen et al. [27].

MD simulations by Yuri and Okamoto, assuming the S-LSR lattice [28,29], suggest that it is possible to generate

\footnotetext{
${ }^{1}$ In this regard, the S-LSR lattice has a relatively good beamdynamic feature compared to the lattices of other cooler storage rings. The vertical phase advance in the cooling mode is actually below 90 degrees $\left(\sim 66.3^{\circ}\right)$, which implies that no serious heating due to linear resonance crossing takes place in lasercooling experiments at S-LSR. The horizontal phase advance $\left(\sim 124.1^{\circ}\right)$ is twice higher, but it still satisfies the maintenance condition [12]. Refer to [28] in detail.
} 
an ultralow emittance ion beam in S-LSR. It has been concluded that a beam phase transition to 1D string or 2D zigzag is feasible by the conventional Doppler cooling technique, provided that a sufficiently powerful laser is employed together with the SBRC technique. The production of a 3D crystalline beam further requires the so-called tapered cooling $[12,30]$ or a combination of dispersionfree bending elements [31] and a coupling cavity [10,32], in order to avoid shear heating.

At relatively high beam intensity as considered here, the transverse cooling efficiency is crucially important. Too low a transverse cooling rate simply makes it impossible to overcome various heating sources including Coulomb collisions (IBS), space-charge-induced collective resonances, lattice-imperfection-induced resonances, and the vertical instability pointed out by Madsen et al. In the MD study cited above [29], the total number of ${ }^{24} \mathrm{Mg}^{+}$ions in S-LSR is only about a thousand, 4 orders of magnitude smaller than the present experiment. With $10^{7}$ ions in the ring, Coulomb collisions strongly affect the attainable beam temperature as demonstrated in Fig. 10. It might, therefore, be interesting to see what happens when we reduce the beam intensity suppressing heating from IBS and collective instability mechanisms. Systematic SBRC experiment are now in progress at S-LSR with much fewer initial ions. This is, however, out of the scope of the present paper and will be reported in a forthcoming paper.

To conclude, we have succeeded in confirming the enhancement of the horizontal laser-cooling rate by means of the SBRC method for the first time at the storage ring S-LSR. Although the cooling time is still rather long due to the limited laser power and strong IBS heating, a significant reduction of the horizontal beam size is confirmed through laser-induced fluorescence measurements when the synchrotron and betatron tunes satisfy the SBRC condition in Eq. (1). The initial horizontal temperature of $\sim 6000 \mathrm{~K}$ has been reduced to $\sim 200 \mathrm{~K}$ after the indirect transverse cooling. Unlike the sympathetic cooling scheme that essentially relies on natural Coulomb collisions, the SBRC scheme develops an artificial (thus controllable) transverse cooling force.

\section{ACKNOWLEDGMENTS}

This work was supported by Advanced Compact Accelerator Development Project of MEXT and the Global COE program "The Next Generation of Physics, Spun from Universality and Emergence."

[1] G. I. Budker, Sov. At. Energy 22, 346 (1967) [http://link .springer.com/article/10.1007/BF01175204].

[2] D. Möhl, G. Petrucci, L. Thorndahl, and S. van der Meer, Phys. Rep. 58, 76 (1980).

[3] D. J. Wineland and H. Dehmelt, Bull. Am. Phys. Soc. 20, 637 (1975).
[4] T. Hänsch and A. Schawlow, Opt. Commun. 13, 68 (1975).

[5] S. Schröder et al., Phys. Rev. Lett. 64, 2901 (1990).

[6] J.S. Hangst, M. Kristensen, J. Nielsen, O. Poulsen, J. Schiffer, and P. Shi, Phys. Rev. Lett. 67, 1238 (1991).

[7] J.S. Hangst, J.S. Nielsen, O. Poulsen, P. Shi, and J. P. Schiffer, Phys. Rev. Lett. 74, 4432 (1995).

[8] H. J. Miesner, R. Grimm, M. Grieser, D. Habs, D. Schwalm, B. Wanner, and A. Wolf, Phys. Rev. Lett. 77, 623 (1996).

[9] N. Madsen, P. Bowe, J. S. Nielsen, L. E. Siegfried, and J. S. Hangst, Phys. Rev. Lett. 87, 274801 (2001).

[10] H. Okamoto, A. M. Sessler, and D. Möhl, Phys. Rev. Lett. 72, 3977 (1994).

[11] H. Okamoto, Phys. Rev. E 50, 4982 (1994).

[12] J. Wei, H. Okamoto, and A. M. Sessler, Phys. Rev. Lett. 80, 2606 (1998).

[13] I. Lauer, U. Eisenbarth, M. Grieser, R. Grimm, P. Lenisa, V. Luger, T. Schätz, U. Schramm, D. Schwalm, and M. Weidemüller, Phys. Rev. Lett. 81, 2052 (1998).

[14] U. Schramm and D. Habs, Prog. Part. Nucl. Phys. 53, 583 (2004).

[15] T. Schätz, U. Schramm, and D. Habs, Nature (London) 412, 717 (2001).

[16] U. Schramm, T. Schätz, and D. Habs, Phys. Rev. Lett. 87, 184801 (2001).

[17] A. Noda, Nucl. Instrum. Methods Phys. Res., Sect. A 532, 150 (2004).

[18] A. Noda, M. Ikegami, and T. Shirai, New J. Phys. 8, 288 (2006).

[19] H. Souda et al., Nucl. Instrum. Methods Phys. Res., Sect. A 597, 160 (2008).

[20] M. Tanabe, T. Ishikawa, M. Nakao, H. Souda, M. Ikegami, T. Shirai, H. Tongu, and A. Noda, Appl. Phys. Express 1, 028001 (2008).

[21] A. Noda et al., in Proceedings of COOL '07 (Digitaldruck Leibi.de, Neu-Ulm, Germany, 2007), p. 221.

[22] H. Souda et al., in Proceedings of the International Particle Accelerator Conference, Kyoto, Japan (ICR, Kyoto, 2010), p. 861.

[23] W. Petrich et al., Phys. Rev. A 48, 2127 (1993).

[24] J. S. Hangst, A. Labrador, V. Lebedev, N. Madsen, J. Nielsen, O. Poulsen, P. Shi, and J. Schiffer, Phys. Rev. Lett. 74, 86 (1995).

[25] N. Madsen, P. Bowe, M. Drewsen, L. Hornekær, N. Kjærgaard, A. Labrador, J. Nielsen, J. Schiffer, P. Shi, and J. Hangst, Phys. Rev. Lett. 83, 4301 (1999).

[26] V. V. Parkhomchuk and D. V. Pestrikov, Sov. Phys. Tech. Phys. 25, 818 (1980).

[27] N. Madsen, P. Bowe, M. Drewsen, L. H. Hornekær, N. Kjærgaard, A. Labrador, J. S. Nielsen, J. P. Schiffer, P. Shi, and J. S. Hangst, Nucl. Instrum. Methods 441, 203 (2000).

[28] Y. Yuri and H. Okamoto, Phys. Rev. ST Accel. Beams 8, 114201 (2005).

[29] Y. Yuri and H. Okamoto, Phys. Rev. Lett. 93, 204801 (2004).

[30] H. Okamoto and J. Wei, Phys. Rev. E 58, 3817 (1998).

[31] M. Ikegami, A. Noda, M. Tanabe, M. Grieser, and H. Okamoto, Phys. Rev. ST Accel. Beams 7, 120101 (2004).

[32] T. Kihara, H. Okamoto, Y. Iwashita, K. Oide, G. Lamanna, and J. Wei, Phys. Rev. E 59, 3594 (1999). 